Prevalence of MG was 18\%. Of MG-positive FSW, $18 \%$ were coinfected with chlamydia or trichomonas respectively, 9\% with gonorrhoea. Diagnosis of MG was associated with younger age $(\mathrm{aOR}=0.97 ; 95 \mathrm{CI}=0.95-0.99$, per year increasing $)$, being born in Bulgaria $(\mathrm{aOR}=2.9 ; 95 \mathrm{CI}=1.2-7.2)$ or Romania $(\mathrm{aOR}=3.4 ; 95 \mathrm{CI}=1.4-8.3$; reference Germany $)$, having no German skills $(\mathrm{aOR}=3.1 ; 95 \mathrm{CI}=1.2-8.0)$, having no health insurance $(\mathrm{aOR}=1.8 ; 95 \mathrm{CI}=1.0-3.2)$, and an interaction term of German skills and health insurance status.

Conclusion Prevalences of MG and STI-coinfections were high in the tested population, especially in younger, migrated FSW, without German skills and health insurance. Tests for MG, and if tested positive, for chlamydia, trichomonas and gonorrhoea, should be offered for FSW, combined with cultural mediation. Disclosure of interest statement The testkits and reagents used in the scope of the study were distributed free of cost by Hologic.

\section{P09.07 PREVALENCE OF MYCOPLASMA GENITALIUM IN PATIENTS VISITING HIV COUNSELLING INSTITUTIONS IN NORTH-RHINE-WESTPHALIA, GERMANY (STI-HIT STUDY)}

\footnotetext{
${ }^{1,2} \mathrm{~A}$ Lallemand, ${ }^{1} \mathrm{~V}$ Bremer, ${ }^{1} \mathrm{~K}$ Jansen* ${ }^{*},{ }^{1} \mathrm{~S}$ Nielsen, ${ }^{3} \mathrm{D}$ Münstermann, ${ }^{3} \mathrm{~A}$ Lucht, ${ }^{3} \mathrm{C}$ Tiemann. ${ }^{1}$ Robert Koch Institute, Berlin, Germany; ${ }^{2}$ European Programme for Intervention Epidemiology Training (EPIET), ECDC, Stockholm, Sweden; ${ }^{3}$ Labor Krone, Bad Salzuflen, Germany
}

\subsection{6/sextrans-2015-052270.391}

Introduction Patients asking for a free anonymous HIV test may have other STI such as Mycoplasma genitalium $(\mathrm{Mg})$, yet $\mathrm{Mg}$ prevalence in that population is unknown. Among other STI, we measured $\mathrm{Mg}$ prevalence in patients seeking HIV testing at local public health authorities (LPHA) to inform decision making regarding $\mathrm{Mg}$ screening in HIV counselling institutions.

Methods LPHA in North Rhine-Westphalia screened patients for $\mathrm{Mg}$ infection on the basis of self-collected vaginal swabs and urine samples (men) which were analysed by transcription-mediated amplification assays (APTIMA ${ }^{\circledR}$ Mycoplasma genitalium). LPHA staff collected information on socio-demographic characteristics, sexual and HIV testing behaviours, previous STI history and clinical symptoms. We assessed overall and group-specific $\mathrm{Mg}$ prevalences and their 95\% confidence intervals (CI). Using binomial regression, we calculated prevalence ratios adjusted for age and level of education.

Results Eighteen LPHA collected 3204 samples, of which seventeen could not be analysed. Of the remaining 3187 samples, LPHA recruited 1751 men, 1430 women and 6 transgenders. The median age was 30 years [25-38]. Overall prevalence of $\mathrm{Mg}$ was 3.42\% (109/3187; 95\% CI: $2.8-4.1 \%), 72.5 \%$ of those who tested positive were asymptomatic. $\mathrm{Mg}$ prevalence was 1.4\% (16/1174; 95\% CI: $0.8-2.2 \%)$ in heterosexual men, $2.0 \%$ (11/549; 95\% CI: 1.0-3.6\%) in MSM, $13.6 \%$ (39/286; 95\% CI: 9.9-18.2\%) in female sex workers (FSW) and 3.6\% (41/1144; 95\% CI: $2.6-4.8 \%)$ in other women. After adjusting for age and level of education, $\mathrm{Mg}$ infection was 7.4 times [95\% CI: 4.0-13] and 2.8 times [95\% CI: $1.5-5.0]$ more prevalent in FSW and in other women, respectively, as compared to heterosexual men. Conclusion The prevalence of $\mathrm{Mg}$ was higher in women, especially among FSW. Use of vaginal swabs in women as compared to urine samples in men may have provided more sensitive detection of $\mathrm{Mg}$ among women. Increased attention should be paid to $\mathrm{Mg}$ screening in patients seeking HIV testing at LPHA, particularly among FSW.
Disclosure of interest statement Diagnostic tests were funded by Hologic $^{\circledR}$.

\section{P09.08 STRONG AND ONGOING INCREASE OF SYPHILIS IN MSM IN GERMANY}

K Jansen*, V Bremer. HIV and STI Unit, Robert Koch-Institute, Berlin, Germany

10.1136/sextrans-2015-052270.392

Background In Germany, the number of syphilis cases doubled 2001-04 to over 3,000/year and remained mainly stable until 2009. Between 2010 and 2013, the number increased between $11 \%$ and $22 \%$. We analysed syphilis surveillance data to assess characteristics of this rise and whether it is continuing.

Methods Since 2001, laboratories are required to notify syphilis diagnoses anonymously, physicians complement clinical information. Potential double notifications are identified. We described syphilis cases by year of diagnosis, age, sex, area of residence and transmission category.

Results Overall, 5,722 cases were reported in 2014, corresponding to a $14 \%$ rise compared to 2013 . The overall incidence was 7.1 per 100,000 inhabitants, with highest incidences in large cities such Berlin (31.0), Cologne (31.9) and Munich (27.2), especially in Berlin inner city areas (61.3-86.2/100,000 inhabitants). Notified cases increased in 13/16 federal states in 2014, and increase in Germany in a linear manner by 669 cases/year since $2010\left(\mathrm{R}^{2}=0.9994\right)$.

Men accounted for $94 \%$ of cases in 2014. The likely mode of transmission was available for $74 \%$ of cases; of these, $84 \%$ were men who have sex with men (MSM), in 16\% heterosexual transmission was reported. The proportion of MSM aged 40 years or above was stable since 2008 with 49\%. Stage of infection was reported in $74 \%$ of cases in 2014 . Of these, $35 \%$ were diagnosed as primary, $27 \%$ as secondary and $35 \%$ as latent syphilis, similar to previous years.

Conclusion Syphilis cases continue to increase strongly since 2010, mainly attributable to MSM in large German cities, also in higher age groups. Berlin as a centre of sex tourism for MSM worldwide is heavily affected. Against the background of high proportions of cases diagnosed in later stages, early diagnosis and treatment, but also consistent condom use, are important to minimise the risk of syphilis and subsequently potential HIVtransmission.

Disclosure of interest statement The authors have nothing to disclose.

\section{P09.09 EXPLORING THE RELATIONSHIP BETWEEN IMPORTATION FREQUENCY AND THE PERSISTENCE OF GONORRHOEA STRAINS IN AN MSM POPULATION: A MODELLING STUDY}

${ }^{1} \mathrm{~B} \mathrm{Hui}^{*}{ }^{2}{ }^{2} \mathrm{D}$ Whiley, ${ }^{1,3} \mathrm{~B}$ Donovan, ${ }^{1} \mathrm{D}$ Regan, on behalf of GRAND Study Investigators. ${ }^{1}$ The Kirby Institute; ${ }^{2}$ QPID Laboratory, QCMRI, The University of Queensland; ${ }^{3}$ Sydney Sexual Health Centre

\subsection{6/sextrans-2015-052270.393}

Introduction Molecular typing of fluoroquinolone resistant isolates from the US suggests that the importation of new strains, rather than mutation within existing strains, is the main source of Neisseria gonorrhoeae (NG) antimicrobial resistance (AMR) emerging within a properly treated population. In Australia, 